\title{
La investigación sobre los flujos de información en las fábricas: el enfoque de la disciplina administrativa
}

Information flows in factories: a state of the question of the perspective of Management Science

\section{Celso Martínez Musiño}

Universidad Nacional Autónoma de México, México celsommm@yahoo.com.mx

\begin{abstract}
Resumen
Se realiza un estado de la cuestión de los estudios de tipo paradigmático y pragmático acerca de los flujos de información en las fábricas desde la perspectiva de la ciencia administrativa. Se localizaron y analizaron siete investigaciones publicadas en revistas académicas en el periodo 1959-2006. Se ha identificado que en todas las áreas sustantivas de las organizaciones manufactureras se utilizan y produce información, concentrándose en funciones administrativas. A las fuentes de información interna se les suman como fuentes de información los clientes, los proveedores, la competencia e incluso las redes sociales.
\end{abstract}

Palabras clave: Flujos de información. Sector secundario. Fábricas. Ciencia de la administración.

\section{Introducción}

Esta investigación tiene su antecedente en la tesis Los flujos de información en las organizaciones: análisis de los enfoques de la administración y la ciencia de la información (Martínez Musiño, 2012b) cuyo objetivo fue indagar las investigaciones que abordaban la fenomenología de la información en las organizaciones manufactureras. Parte de los hallazgos encontrados, en relación con la ciencia de la información, se publicaron en el artículo Tipificación y uso de los recursos de información en las organizaciones manufactureras (Martínez Musiño, 2011b). Cabe mencionar que en este artículo se localizaron, recuperaron y analizaron solo artículos académicos de los siguientes autores: Bergeron (1997), Chan (2001), Mbambo \& Cronjé (2002), Verville \& Halingten (2003), Almeida Moraes, Giseli Diniz \& Escrivão Filho (2006), De Saulles (2007) y Choo, Bergeron, Detlor \& Heaton (2008). Una de las conclusiones de los estudios sobre la información en las fábricas, desde el punto de vista particular de la ciencia de la información es que es un tema reciente, como demuestra la cantidad de artículos. Otra conclusión es que quienes hacen uso de la informa-

\begin{abstract}
A state of the art of the paradigmatic and pragmatic research on information flows in factories from a Management Science approach are analyzed. Seven studies published in academic journals in the period 1959-2006 were located and analyzed. All the substantive areas in factories produce and use information, mostly the administrative functions. Besides internal information sources, factories use other ones, remarkably customers, suppliers, competitors, and even social networks.
\end{abstract}

Keywords: Information flows. Secondary sector. Factories. Management science.

ción en las organizaciones manufactureras son los directivos.

Sin embargo, la escases de estudios académicos de la fenomenología de la información en esta área del conocimiento, nos motivó a ampliar el espectro, en concreto a la disciplina administrativa.

La disciplina administrativa ha encontrado argumentos en los cuales la información se ha erigido como motor de cambios en distintas etapas del desarrollo humano. Peter F. Drucker, considerado pilar de la administración moderna, identifica y fundamenta esta afirmación de la manera siguiente: a) la información es una herramienta; b) los gerentes de las organizaciones hacen un uso importante de la información; c) la información es un recurso clave de los ejecutivos; d) la información es pieza clave de la revolución informática en los negocios; e) la información es valorada como la columna vertebral de las organizaciones; y f) el valor de la información trasciende dentro y fuera de las organizaciones (Drucker, apud Martínez Musiño, 2012a). En concordancia con Drucker, en la consideración de la información como recurso clave para la administración de las organizaciones, coinciden tanto John P. Kotter como Harold 
Koontz \& Cyril O’Donnell. Por un lado, Kotter (1984, p. 9) afirma que, "en el proceso de reunir información necesaria para establecer sus agendas, los gerentes generales se basan más en sus pláticas con otros que en los libros, revistas o informes", mientras que Koontz \& O'Donell hacen alusión al exceso de información como una preocupación de los ejecutivos, al considerar que "la información puede contener cualquier cosa, desde los más determinado y minucioso hasta lo universal" (1970, p. 610).

A las aportaciones de Drucker, Kotter y Koontz \& O'Donell, se le pueden sumar las de Mintzberg (1992), quien no solo encontró que la información tiene un valor particular en las organizaciones, sino que descubrió que los directivos ejercen ciertos roles informacionales (Martínez Musiño, 2013).

Ante las evidencias del enfoque administrativo sobre la importancia de la información en las organizaciones, es posible cuestionarse si la información es producida o utilizada, no solo para las actividades gerenciales, sino para otros procesos como el control de compras de materias primas o la generación de ideas para la innovación, tanto para nuevos productos como para la reorganización de procesos existentes.

La organización y utilización adecuada de la información en las organizaciones posibilita un aprendizaje tanto en los individuos como en los equipos de trabajo. Este aprendizaje puede generar conocimientos; y éstos, a su vez, producen beneficios para la organización, como la innovación y el enriquecimiento de experiencias. En todas las organizaciones, incluidas las manufactureras, se genera información, tanto de manera individual como colectiva. Las organizaciones manufactureras, que tienen como meta principal adquirir materias primas, transformarlas y venderlas, también se encuentran en un contexto de economía globalizada; y su permanencia en el mercado implica la necesidad de innovar, producir más con menores recursos y aprender de la competencia.

En este sentido, vale la pena preguntarse, qué papel juega la información y el conocimiento colectivos. En las organizaciones hay flujos de información que originan conocimiento mediante la interacción de sus integrantes. En consecuencia, el interés por estudiar los flujos de información se debe a que éstos han adquirido importancia para la toma de decisiones. Erik R. Peterson (2004) proyecta que los flujos de información son una de las siete revoluciones en la estrategia global para el año 2025, y su razón principal radica en el uso que se le da a la información en distintos ámbitos para ayudar a la toma de mejores decisiones, según añade la revista The Manager's Electronic Resource Center en el artículo Transporte y uso de la información en su organización (Moving and using information in your organization, 2006).

Después de externar los antecedentes de la investigación, los problemas a los que se enfrentan las organizaciones y los motivos para realizar el estudio, es necesario definir el objetivo principal: buscar y analizar los estudios que examinan los flujos de la información en las fábricas desde el enfoque de la disciplina administrativa. Para cumplir con el objetivo propuesto, el trabajo se ha estructurado en cinco capítulos: 1) Administración y flujos de información: conceptos; 2) Método y procedimiento; 3) Las investigaciones de los flujos de información en las fábricas (resultados); 4) Discusión; y 5) Conclusiones.

\section{Administración y flujos de información: conceptos}

La administración, disciplina eminentemente práctica, es definida por varios autores como un conjunto de procesos compuesto por la planeación, la organización, la dirección y el control mediante los cuales es posible cumplir metas comunes (Bartol \& Martin, 1998, p. 711; Daft \& Marcic, 2006, p. G-1; Jones \& George, 2008, p. 746; Jones, George \& Hill, 2000, p. 720; Higgins, 1991, p. G-10; Kinicki \& Williams, 2006, p. G7).

Por otra parte, a los flujos de información, Gabarro \& Kotter (1992, p. 236-237) los conceptualizan como aquello que necesitan los jefes para saber qué es lo que hacen sus subordinados. Esta definición es un tanto limitada, en la medida en que solo denota dos agentes: los jefes y los subordinados, pero ¿Dónde está el uso de la información por parte de los subordinados? es decir, ¿Hacia dónde se dirige la organización? ¿Cuáles son su misión, objetivo y metas?

Otro término relacionado con los flujos de información es compartir información, que, según Robbins (2000, p. 46), se refiere a que la información es poder; por lo tanto, ésta debe ser administrada y guardada cuidadosamente. En contraposición a los estudios recientes, esta idea de cuidar celosamente la información ha desaparecido: Ahora es más común que los integrantes de cualquier organización sepan a qué se dedica empresa en la cual laboran, cuál es la calidad de los productos o servicios esperados, o qué actividades realizan los integrantes de otras secciones, por mencionar algunos. 
Respecto a las definiciones de flujo de información y compartir información, consignadas en el párrafo anterior, no se está totalmente de acuerdo. Si bien es cierto que la información es producida y utilizada por los integrantes de la cúpula organizacional, se infiere que también cualquier miembro de la organización es productor de información. Si bien, el concepto flujos de información en la disciplina administrativa no se define como concepto, la información sí ha encontrado eco en las definiciones de la administración misma. Por ejemplo, hay vestigios documentales en los cuales se describen a los datos como el origen de la información, la información en la toma de decisiones en incluso se han acuñado los roles informacionales para el trabajo de los directivos (Martínez Musiño, 2013, p. 8-9).

\section{Método y procedimiento}

El estado de la cuestión se realizó según el siguiente procedimiento. Primero, se realizaron búsquedas y recuperación de información en bases de datos especializadas (ISI Web of science), servicios de acceso a bases de datos en humanidades y ciencias sociales (Ebsco, Swets, Wilson, CLASE, PERIODICA, INFOBILA, Latindex, SciELO) y catálogos institucionales de educación superior e investigación (Library of Congress, El Colegio de México, UNAM, UAM, Universidad de Zaragoza, The european library). La configuración de búsqueda para recuperación de registros bibliográficos en la base de datos ISI Web of Science se filtró con la opción "All databases", desde el periodo 1900 al 5 de enero de 2012, y se realizaron las siguientes búsqueda: 1) Topic: Information and flow and organizations; 2) Topic: Information and flow and factories; 3) Topic: Information and flow and manufacture. Finalmente, como complemento a la búsqueda de información en el ISI Web of science, se procedió a la exploración de referencias y artículos de texto completo en las bases de datos especializadas en ciencias sociales y ciencia de la información de Ebsco, Swets y Wilson.

La presentación de resultados se organizó en dos grandes bloques: a) los estudios paradigmáticos, en los cuales se contemplan aquellos que se refieren a lo que deben ser los flujos de información y la descripción de sus ventajas, y; b) investigaciones pragmáticas, en las incluyen estudios de carácter empírico. Finalmente, se procedió a la redacción de los resultados, la discusión y las conclusiones.

\section{Las investigaciones de los flujos de información en las fábricas}

Como producto de la búsqueda de información, tanto en bases de datos especializadas como en catálogos de instituciones de educación superior e investigación, se recuperaron, seleccionaron y analizaron 7 artículos publicados en diversas revistas académicas, que se relacionan en la Tabla I.

\begin{tabular}{ll}
\hline Revista & Autor (año) \\
\hline $\begin{array}{l}\text { Academy of Management } \\
\text { Journal }\end{array}$ & Albaum (1964) \\
\hline $\begin{array}{l}\text { California Management } \\
\text { Review }\end{array}$ & Albaum (1967) \\
\hline $\begin{array}{l}\text { Entrepreneurship and } \\
\text { Regional Development }\end{array}$ & Kristiansen, Kimeme \& \\
\hline $\begin{array}{l}\text { International Journal of } \\
\text { Information Management }\end{array}$ & Kudyba (2006) \\
\hline Management Science & Goldman (1959) \\
\hline Management Science & Baker \& Freeland (1972) \\
\hline Production Planning \& Control & Dimitriadis \& Koh (2005) \\
\hline
\end{tabular}

Tabla I. Títulos de revistas académicas y autores analizados (Martínez Musiño, 2012b)

En relación con la fecha de publicación de los autores de la disciplina administrativa, quienes primero publicaron sus obras en un periodo temprano fueron Goldman (1959), Albaum (1964; 1967) y Baker \& Freeland (1972); y, posteriormente, Kristiansen, Kimeme \& Wahids (2005), Dimitriadis \& Koh (2005) y Kudyba (2006). Es factible observar que durante más de treinta años no se publicaron estudios exclusivamente dedicados a la fenomenología de la información en las organizaciones manufactureras. Sin embargo, es posible inferir que el interés por el estudio en esta temática se manifiesta de nuevo a mediados de la primera década del Siglo XXI (Figura 1).

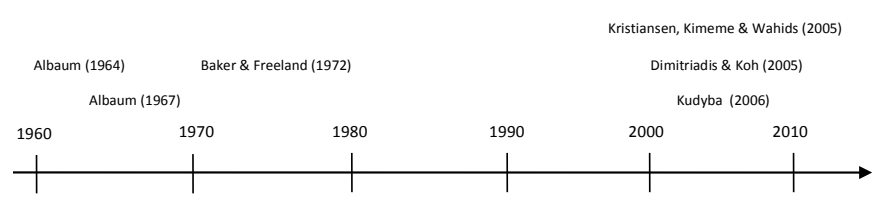

Figura 1. Los autores de los estudios en la línea del tiempo (Martínez Musiño, 2012b)

La descripción detallada de los resultados se presenta según dos categorías: paradigmático y 
pragmático. Al interior de estas dos categorías, se incluyen dos tipos de documentos: a) aquellos en los que el tema principal son las organizaciones manufactureras y en segundo término la información; y, b) aquellos en los cuales se expresan, específicamente, los flujos de información (Tabla II).

\begin{tabular}{lll}
\hline & Administración & \\
\hline $\begin{array}{l}\text { Temas o tipo de } \\
\text { investigación }\end{array}$ & Paradigmático & Pragmático \\
\hline $\begin{array}{l}\text { Organizaciones } \\
\text { manufactureras e } \\
\text { información }\end{array}$ & $\begin{array}{l}\text { Goldman } \\
(1959)\end{array}$ & No disponible \\
& Albaum (1967) & \\
Baker \& & Freeland & \\
& $(1972)$ & \\
\hline $\begin{array}{l}\text { Flujos de la } \\
\text { información en las } \\
\text { organizaciones } \\
\text { manufactureras }\end{array}$ & No disponible & $\begin{array}{l}\text { Albaum (1964) } \\
\text { Dimitriadis \& } \\
\end{array}$ \\
& & $\begin{array}{l}\text { Koh (2005) } \\
\text { Kristiansen, } \\
\end{array}$ \\
& & Kimeme \& \\
& & Wahids (2005) \\
& & Kudyba (2006) \\
\hline
\end{tabular}

Tabla II. Organización temática de los resultados

\subsection{Estudios paradigmáticos}

Se detectaron tres tipos de investigaciones paradigmáticas $y$, según la temática descrita, pueden agruparse de manera general en: 1) flujos de información e innovación (flujo de ideas, recursos de información, investigación administrativa); 2) flujos de información y productividad de los trabajadores (flujo circular de la información operadores-supervisores-grupos de servicios de ingeniería); y, 3) flujos de información y descentralización de la toma de decisiones en el marketing (flujo de información vertical, flujo de información horizontal, y participación e interacción de centros de información con unidades de toma de decisiones). A continuación se presenta la descripción específica de los siete estudios.

i) La investigación más antigua se publicó en los años cincuenta por Alan S. Goldman (1959) quien es el autor del artículo Los flujos de información y la productividad de los trabajadores (en inglés: Information flow and worker productivity). El investigador se propone diseñar una organización en el área de la toma de decisiones mediante el enfoque de la teoría general de sistemas. Goldman describe una estructura piramidal en la cual las organizaciones se componen de un administrador general, el administrador de producción, el jefe, los supervisores y, finalmente, los operadores. De esta estructura, Goldman profundiza en tres elementos: los operadores, los supervisores y un grupo supervisor de ingeniería. En su análisis nos indica que los operadores se encargan de recolectar los datos, mientras que los supervisores elaboran un análisis breve; seguidamente, los supervisores reciben, analizan y envían al grupo supervisor. Gráficamente, la comunicación de estos elementos puede visualizarse en la Figura 2. Alan S. Goldman no hace referencia a alguna tipificación o descripción de la información. Lo rescatable del análisis del autor es la conformación de un flujo de información a partir de los datos, el involucramiento de algunos actores de las organizaciones en la detección de problemas y la propuesta de mejor toma de decisiones, particularmente por un grupo superior de ingeniería.

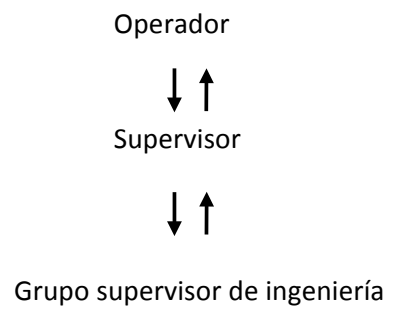

Figura 2. Participación de grupo supervisor de ingeniería en los flujos de información en las organizaciones (elaboración propia con datos de Goldman, 1959)

ii) Posteriormente a la investigación de Goldman, en los años sesenta, Gerald Albaum (1967) publica el artículo: Los flujos de la información y la toma de decisiones descentralizada en el marketing (en inglés: Information flow and descentralized decisión making in marketing) cuyos objetivos no se identifican con mucha claridad, sin embargo, se infiere que su propósito es analizar los flujos de información en los procesos de marketing. El área de Marketing está compuesta por un ejecutivo y cuatro unidades: publicidad y promoción, información, mercadotecnia y administración de ventas. A su vez, de la unidad de información dependen un área de investigación y otra de inteligencia; mientras que de la administración de ventas se desprenden las oficinas y los vendedores.

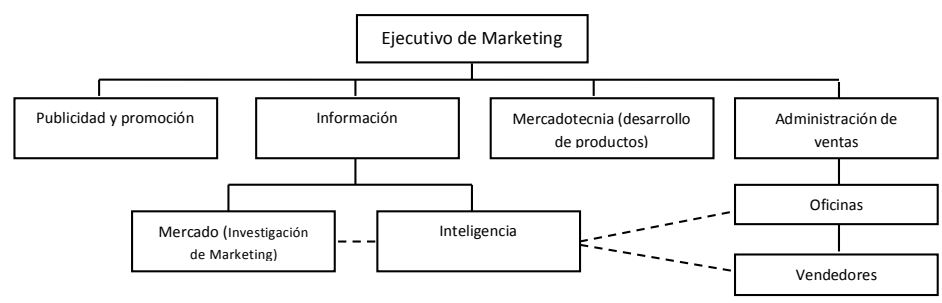

Figura 3. Organización funcional de marketing reflejando el concepto de información total (Albaum, 1967, p. 63) 
Lo rescatable del análisis de Albaum es la integración de una unidad de información que, mediante los servicios de investigación e inteligencia, funge como colectora de información de las oficinas de ventas y los vendedores. En las distintas propuestas analíticas, Albaum, reincidentemente, esquematiza centros de información interactuando con otras unidades de decisión (Figura 3).

iii) En los años setenta, Norman R. Baker \& James R. Freeland (1972, p. 105) publican el artículo Estructurando los flujos de información para mejorar la innovación (en inglés: Structuring information flow to enhance innovation), en el cual se proponen como objetivo discutir el impacto de los flujos de información para la cantidad y la calidad de entrada (inputs) de ideas y para el desarrollo de un sistema de administración de información del comportamiento innovador (idea generation and submission). Los autores, como producto de una investigación empírica en las organizaciones de investigación industrial, describen un modelo de sistema de información, en el cual analizan el rol de la información tanto en la creatividad, como en la generación de ideas (Figura 4).

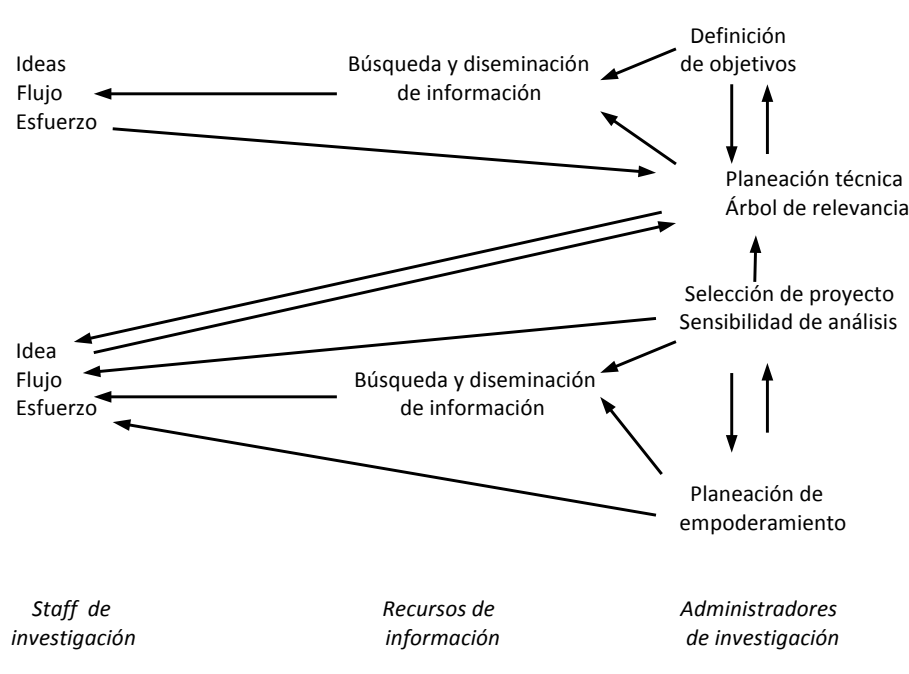

Figura 4. Modelo de sistema de información (Baker \& Freeland, 1972, p. 115)

Baker \& Freeland describen su Modelo partiendo de tres elementos: un staff de investigación, los recursos de información y los administradores de investigación. Estos elementos interactúan en distintos momentos. En primer lugar, se definen los objetivos; posteriormente, se inicia un proceso de búsqueda y diseminación de información; los resultados de ésta generan un primer conjunto de ideas; seguidamente, las ideas pasan a la sistematización (árbol de rele- vancia) para su planeación técnica; y, finalmente, se verifica si con este procedimiento se cumplen los objetivos deseados; si no es así, se genera un nuevo cumulo de ideas y se sistematizan nuevamente. Sin embargo, se puede hacer una crítica al Modelo de Baker \& Freeland: no hay retroalimentación entre la segunda etapa de generación de ideas con los procesos de análisis y la selección del proyecto, ni tampoco con el empoderamiento de la planeación.

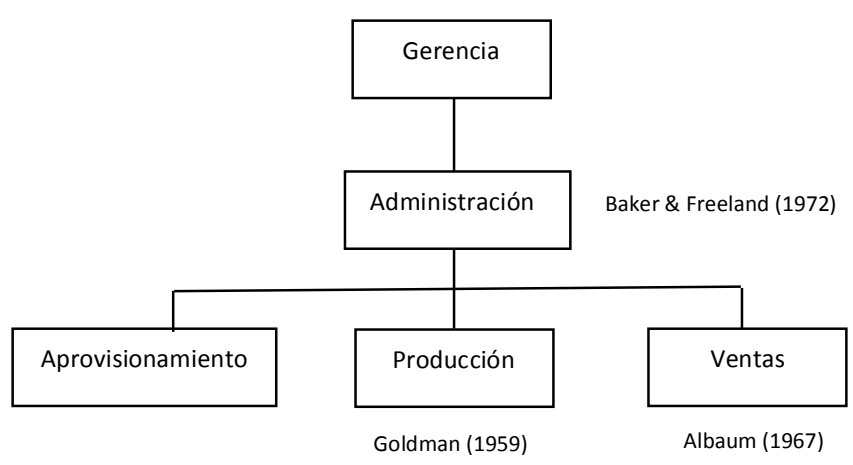

Figura 5. Distribución de los estudios del enfoque administrativo paradigmático en la estructura de las organizaciones manufactureras (Martínez Musiño, 2012b)

Como resumen de esta sección de investigaciones paradigmáticas de las tres investigaciones de la fenomenología de la información en las organizaciones manufactureras se observan las siguientes temáticas: a) la participación de grupos supervisores de ingeniería; b) la inserción del concepto de información total en el marketing; y c) la presentación de un modelo de sistema de información para la innovación. Ahora bien, si las fábricas, en su organigrama más simple, se componen de la gerencia, la administración, el aprovisionamiento de materias primas, la producción y el área de ventas, la distribución de los estudios encontrados se concentra en el área administrativa, el área de producción y el área de ventas; faltan estudios dedicados las áreas de aprovisionamiento y la gerencia (Figura 5).

\subsection{Estudios pragmáticos}

Se localizaron cuatro artículos de caso dedicados a los cuatro temas siguientes: 1) los flujos de la información y la comunicación en las organizaciones manufactureras de componentes eléctricos; 2) los flujos de información y la adaptación y supervivencia de las organizaciones manufactureras del vestido y la madera en Tanzania; 3) los flujos de información y las cadenas 
de proveedores en Kastoria, Grecia; y, 4) los flujos de información y el uso de tecnologías de información en la cadena de aprovisionamiento de materias primas en la industria automotriz de los Estados Unidos de América. A continuación se presenta la descripción particular de las investigaciones encontradas.

iv) La investigación más antigua, años sesenta, es atribuida a Gerald Albaum quien publica el artículo de tipo experimental Flujos de información horizontal: estudio exploratorio (en inglés: Horizontal information flow: an exploratory study), en el cual se propone dos objetivos principales: 1) determinar los canales de flujos de información en una fábrica que produce componentes eléctricos; 2) verificar la distorsión de la información ocurrida dentro de fábrica (Albaum, 1964 , p. 23). Las situaciones problemáticas que describe el autor son fundamentalmente dos: a) las compañías —los potenciales emisores- y usuarios de la información no se conocen respectivamente; b) la información en las organizaciones fluye verticalmente, y es necesario que ésta se distribuya horizontalmente. El autor identifica que el estudio de los flujos de información facilita la toma de decisiones para los fines de la planeación, el desarrollo, el control y la solución de problemas (Albaum, 1964, p. 22).

v) En el estudio de Kristiansen, Kimeme \& Wahids (2005), publicado como artículo y cuyo título es Flujos de información y adaptación en las industrias domésticas en Tanzania (En inglés: Information flow and adaptation in Tanzania cottage industries), estos autores se propusieron como objetivo identificar los canales de flujos de información y su impacto en la adaptación y supervivencia, específicamente en organizaciones manufactureras del vestido y la madera.

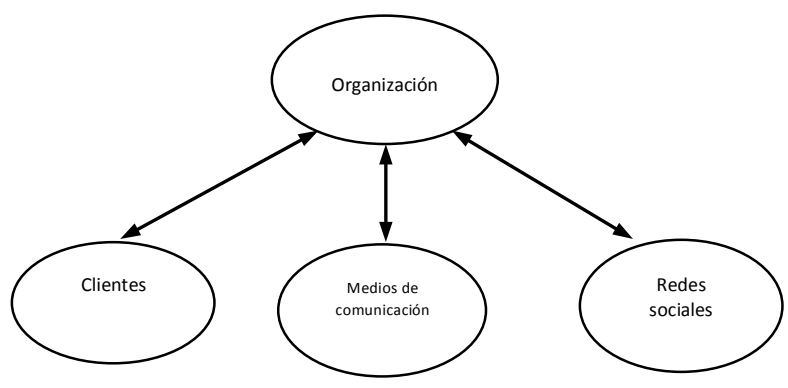

Figura 6. Fuente de información requerida por los administradores de empresas de Kastoria, Grecia (Elaboración propia con datos de Kristiansen, Kimeme \& Wahid, 2005)

Los autores presentan una investigación empírica en la cual aplicaron cuestionarios a 392 or- ganismos en Tanzania. Los encuestados manifestaron utilizar distintas fuentes de información: clientes, medios y redes sociales (familia, colegas y afiliaciones religiosas) (Figura 6).

vi) En la investigación de Dimitriadis \& Koh (2005), Flujos de información y administración de la cadena de suministros en las redes locales de producción: el rol de las personas y los sistemas de información (en inglés: Information flow and supply chain management in local production networks: the role of people and information system), los autores se proponen explorar la administración de cadenas de proveedores en las redes de producción local de las pequeñas y medianas empresas de Kastoria, Grecia. En su disertación, de carácter exploratorio, aplicaron la metodología cualitativa, utilizando como instrumento de colección de datos la entrevista semi-estructurada. Los autores entrevistaron a cuatro empresas líderes de 30, 12 unidades de producción primaria de 30 y 10 unidades de producción de 30 compañías.

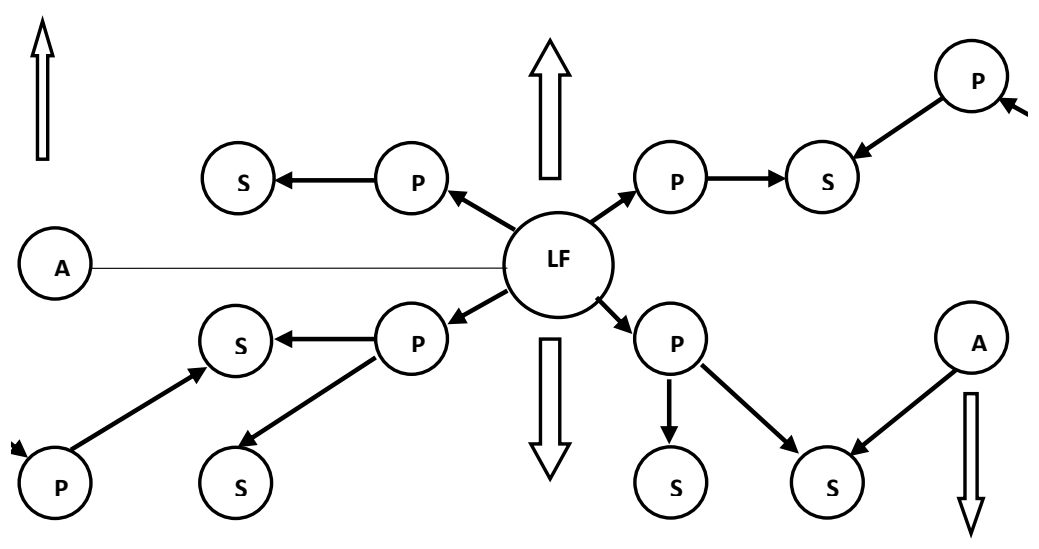

Figura 7. Representación gráfica de las redes de producción locales en Kastoria, Grecia (Dimitriadis \& Koh, 2005)

Las relaciones de estas organizaciones manufactureras se representan en la Figura 7. Los flujos de información caracterizan la cadena de suministro en las organizaciones manufactureras estudiadas (Dimitriades \& Koh, 2005, p. 546). En esta representación gráfica LF significa empresa líder; $\mathrm{P}$, redes de producción primaria; $S$, unidades de producción secundaria; y A, unidades de producción autónomas; las flechas negras representan órdenes de producción; las flechas blancas hacia abajo simbolizan órdenes de materia prima; y las flechas blancas hacia arriba significan bienes terminados para los clientes. Respecto a las unidades de producción de las organizaciones manufactureras, éstas interactúan con los proveedores y los puntos de venta. 


\begin{tabular}{llll}
\hline Externa & $\%$ & Interna & $\%$ \\
\hline Moda en piel & 97 & $\begin{array}{l}\text { Competencia } \\
\text { local }\end{array}$ & 90 \\
\hline $\begin{array}{l}\text { Producción de materias } \\
\text { primas }\end{array}$ & 93 & $\begin{array}{l}\text { Problemas } \\
\text { locales }\end{array}$ & 53 \\
\hline Competencia global & 90 & $\begin{array}{l}\text { Nuevas técnicas } \\
\text { de producción }\end{array}$ & 33 \\
\hline $\begin{array}{l}\text { Legislación/impuestos } \\
\text { locales }\end{array}$ & 80 & $\begin{array}{l}\text { Mano de obra } \\
\text { productiva }\end{array}$ & 17 \\
\hline $\begin{array}{l}\text { Oportunidades externas de } \\
\text { financiamiento }\end{array}$ & 73 & $\begin{array}{l}\text { Iniciativas } \\
\text { colectivas locales }\end{array}$ & 10 \\
\hline Preferencias de los clientes & 67 & & \\
\hline $\begin{array}{l}\text { Avances tecnológicos en } \\
\text { producción }\end{array}$ & 57 & & \\
\hline $\begin{array}{l}\text { Política/economía en } \\
\text { mercados finales }\end{array}$ & 40 & & \\
\hline $\begin{array}{l}\text { Oportunidades para nuevos } \\
\text { mercados }\end{array}$ & 40 & \\
\hline $\begin{array}{l}\text { Legislación/impuestos en } \\
\text { mercados finales }\end{array}$ & 20 & \\
\hline $\begin{array}{l}\text { Tendencias generales en } \\
\text { las modas }\end{array}$ & 10 & \\
\hline $\begin{array}{l}\text { Logística en mercados } \\
\text { finales }\end{array}$ & 10 & \\
\hline $\begin{array}{l}\text { Oportunidades locales de } \\
\text { financiamiento }\end{array}$ & 10 & \\
\hline $\begin{array}{l}\text { Perfil de vendedores en los } \\
\text { mercados finales }\end{array}$ & 7 & \\
\hline
\end{tabular}

Tabla III. Temática requerida por los administradores de las empresas de Kastoria, Grecia, según tipo, externa o interna (extracto de Dimitriadis \& Koh, 2005, p. 550)

Los autores resaltaron también otros resultados de importancia de su investigación: a) el origen de la información se obtiene tanto de manera interna como externa; b) los temas de interés son la información acerca de la competencia y problemas de la localidad, nuevas técnicas de producción, la mano de obra productiva y las iniciativas colectivas locales; c) respecto a los temas de interés externo son: la moda en piel, la producción de materias primas, la competencia global, la legislación y las obligaciones respecto a los impuestos locales, las oportunidades externas de financiamiento, las preferencias de los clientes, y los avances tecnológicos en producción, entre otros.

En un segundo plano de importancia, los temas de interés externos que se localizaron fueron: la política o economía de los mercados finales, la búsqueda de oportunidades de nuevos mercados, la legislación y normatividad regulatoria de los impuestos en los mercados finales, las tendencias generales en la moda, la logística de los mercados finales, las oportunidades de finan- ciamiento y el perfil de los vendedores en los mercados finales (Tabla III, en la página anterior). Finalmente, lo más representativo de los resultados encontrados en esta investigación es que la mayor parte de los flujos de información es mediante las interacciones humanas (Dimitriadis \& Koh, 2005, p 552).

vii) Finalmente, la investigación de Stephan Kudyba, titulada Mejorando los flujos de información organizacional y la creación del conocimiento en la reingeniería de los sistemas de la cadena de aprovisionamiento: un análisis de las piezas de automóviles estadounidenses y el modelo de suministros (en inglés: Enhancing organizational information flow and knowledge creation in re-engineering supply chain systems: an analysis of the U.S. automotive parts and supplies model), tuvo como objetivo direccionar los conceptos esenciales en las cadenas de proveedores y describir el sistema que la industria automotriz de los Estados Unidos de América utiliza (2006, p. 163).

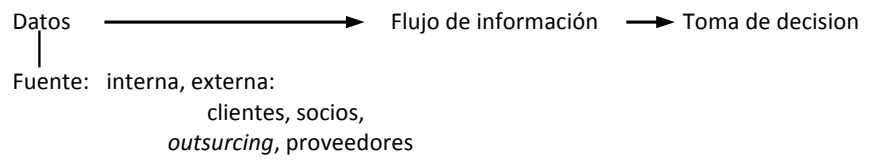

Grupo

supervisor

de ingeniería:

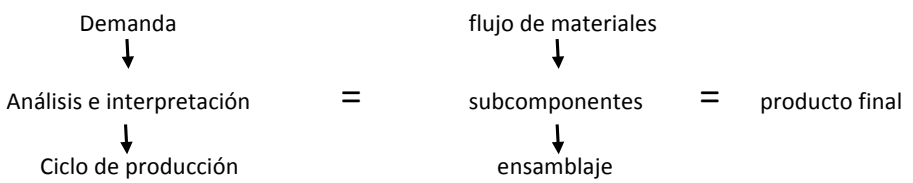

Figura 8. Los flujos de información en las operaciones de la cadena de producción en las organizaciones manufactureras automotrices (elaboración propia con datos de Kudyba, 2006)

La tesis principal del autor es que los datos, su organización y análisis ayudan al flujo de información; y ésta, a su vez, a una mejor toma de decisiones y a la generación de conocimientos. En su estudio, centrado en la cadena de aprovisionamiento de materia prima, el autor señala la necesidad del uso de tecnologías de información que faciliten la captura de datos y su transformación en flujos de información en el proceso de flujo de materiales -es decir, de componentes para su ensamblaje- - y en la generación de productos finales (Figura 8). En el análisis de Kudyba, se menciona la participación de un grupo supervisor de ingeniería, quienes se en- 
cargan de la solicitud de datos de flujos de materiales, su análisis e interpretación y los ciclos de producción.

Como resumen preliminar de la exposición de resultados referente a las investigaciones de carácter empírico, se puede concluir que: 1) los estudios son aún limitados y se focalizan en ciertos sectores de las organizaciones manufactureras, a las unidades de aprovisionamiento de materias primas y la manera en que es comunicada la información; 2) la información se puede obtener, tanto de manera interna como de manera que busque soluciones de permanencia en el mercado, como es el caso de las organizaciones manufactureras textiles de Kastoria, Grecia; 3) los flujos de información corren de manera paralela con el flujo de materiales, su ensamblaje y la terminación de productos, tal es el ejemplo de la investigación de Kudyba (2006). Por otra parte, de acuerdo a los componentes estructurales de las organizaciones manufactureras (la gerencia, la administración, el área de aprovisionamiento de materias primas, la sección de producción y la unidad de ventas), los autores concentran sus investigaciones en algunas áreas específicas, que se presentan visualmente en la Figura 9.

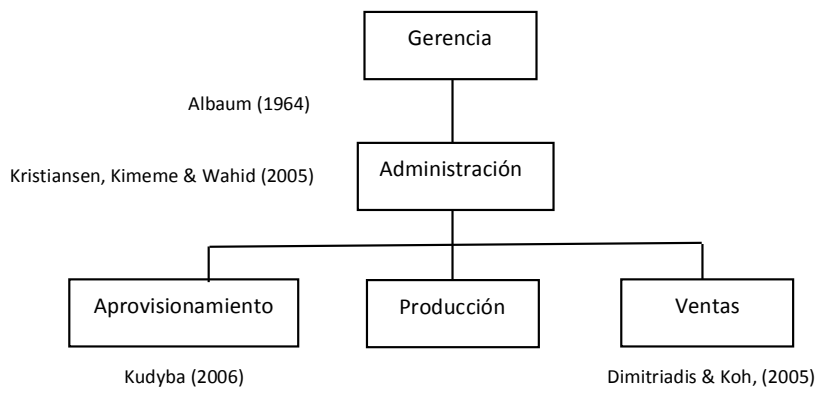

Figura 9. Distribución de los estudios del enfoque administrativo pragmático en la estructura de las organizaciones manufactureras (Martínez Musiño, 2012b)

A diferencia de lo que ocurre en las investigaciones paradigmáticas, este grupo de los autores en sus estudios, prácticamente, abarcaron todas las áreas de las organizaciones manufactureras. Ahora bien, si sumamos tanto los estudios paradigmáticos con los pragmáticos, visualmente obtenemos el panorama que se observa en la Figura 10.

Esto significa que hay mayor concentración de los estudios en las actividades gerenciales y administrativas, seguidas por el área de ventas; y que menor cantidad de investigaciones se centran en las áreas de aprovisionamiento de materias primas y producción.

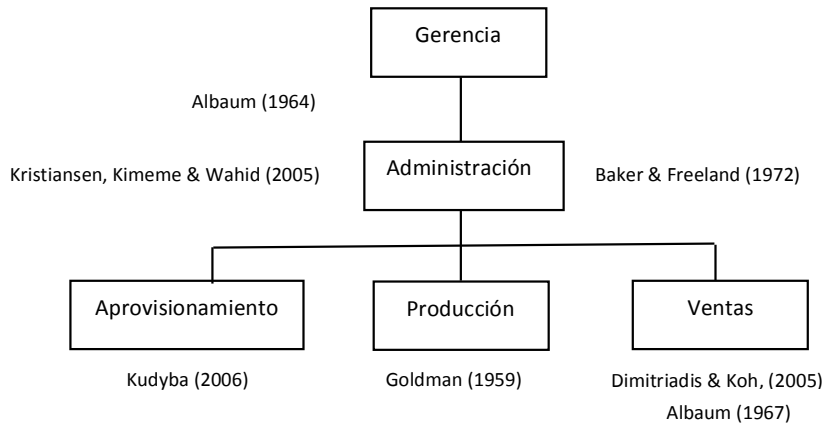

Figura 10. Concentración de los estudios con enfoque administrativo en la estructura de las organizaciones manufactureras (Martínez Musiño, 2012b)

\section{Discusión}

La administración como disciplina eminentemente práctica se ha focalizado en distintos aspectos a lo largo de su historia. Se inició con el énfasis en la organización científica del trabajo. Después, ante la necesidad de hacer de las organizaciones cada vez más rentables, la disciplina administrativa focalizó durante años sus esfuerzos en la motivación de sus recursos humanos. Posteriormente a los recursos materiales, las formas de optimizarlos y combinar estos factores con la búsqueda de mejores condiciones de los individuos, la administración abordó un recurso más: la información.

Al encontrarse vestigios del concepto información y su significado en monografías y artículos en la disciplina administrativa, es perfectamente constatable que la información ha adquirido relevancia significativa. Sin embargo, esta significación no se refleja en el concepto de flujo información, puesto que solo se relaciona con las actividades de los administradores, quienes la utilizan "para saber qué es lo que hacen sus subordinados" (Gabarro \& Kotter, 1992, p. 236237). Sin embargo, los estudios de los flujos de información son necesarios, además, para identificar, tipificar y valorar la manera en que se comparte la información entre los distintos elementos de cualquier organización.

El enfoque administrativo, desde el punto de vista paradigmático, menciona el flujo de información entre varios elementos de las organizaciones: las personas. Por su parte, Albaum menciona la necesidad de unidades de servicios de información (biblioteca, centro de información) para crear sistemas de información. En cambio, desde el punto de vista pragmático, en un primer plano menciona como recursos de información los contenidos en las bibliotecas y aquellos que pueden ofertar los servicios de cómputo. En un segundo plano, el autor considera a la información contenida en las personas 
como sinónimo de ideas al mencionar que los "recursos de información se pueden convertir en parte integral de los flujos de información" (Baker \& Freeland, 1972, p. 113).

De manera particular, la descripción cronológica de la cobertura de la información en las organizaciones manufactureras, según la disciplina administrativa, la investigación de Goldman (1959) se concentra en el diseño e implementación de un área de decisión interna en la cual la información se comparta entre el gerente general y los administradores. Por su parte, Albaum (1964), como primera propuesta, basando su estudio en el problema del flujo de información, hace patente la necesidad de compartir la información de manera vertical para facilitar la toma de decisiones. A diferencia de Goldman (1959), este autor sí incluye a las unidades operativas de la organización para compartir la información.

En su segundo análisis, Albaum (1967) se concentra en una sola actividad de la organización manufacturera: el marketing. Sin embargo, en su propuesta para el uso de la información incluye varios actores, fuentes y recursos de información. Respecto a los actores, el autor menciona las necesidades de los altos ejecutivos, del área de publicidad y promoción, los desarrolladores de productos y los administradores de ventas. Respecto a las fuentes de información, se menciona a los clientes, los proveedores, e incluso los competidores. Finalmente, en relación con los recursos de información, creados internamente, se menciona inclusive se integra en el organigrama- una unidad de información apoyada por centros de información.

Continuando con las particularidades de la cobertura de la información en la disciplina administrativa, Baker \& Freeland (1972) focalizan su investigación en los aspectos intangibles, en este caso en la innovación generada en las organizaciones manufactureras. Los autores, valoran como la información puede incidir en el desarrollo de objetivos organizacionales mediante una unidad de investigación. Por otra parte, en este siglo XXI, Dimitriadis \& Koh (2005) analizan en su investigación los flujos de información y la administración de cadenas de suministro, en los cuales incluyen la participación de los administradores de varias organizaciones manufactureras, apoyados por consultores externos y herramientas tecnológicas para la implementación de sistemas información.

Por otra parte, Kristiansen, Kimeme \& Wahids (2005), al igual que Dimitriadis \& Koh (2005), analizan en su estudio un conjunto de organiza- ciones manufactureras e incluyen como actores principales a los gerentes y administradores. Finalmente, la investigación de Kudyba (2006), al igual que Dimitriadis \& Koh (2005), analiza los flujos de información en la cadena de aprovisionamiento, aunque solo se concentran en organizaciones manufactureras de un país, los Estados Unidos de América.

Después de encontrar un marco referencial teórico y de resultados en los estudios de los flujos de información en las organizaciones manufactureras es posible derivar nuevas preguntas para futuras investigaciones — varias de las cuáles han sido objeto del interés de investigadores en gestión de la información y gestión del conocimiento en otros ámbitos de aplicación y otras se refieren directamente a nuestra línea de investigación-:

- Si la información ha adquirido un valor dentro de las organizaciones, ¿es posible encontrar indicadores? es decir, ¿cómo se mide esa valía?

- En la escala de considerarse a los datos como condición indispensable, entonces ¿cómo la información se transforma en conocimiento?

- Si la información es una herramienta para el trabajo directivo, es decir, para la toma de decisiones, ¿es posible considerar al ser humano como herramienta en tanto que es identificado como portador de información y de conocimiento?

- Si las organizaciones manufactureras se distinguen de otras por transformar la materia prima y crear bienes de consumo, ¿es posible considerar a la información en ese proceso de entrada y salida de materiales y productos terminados?

- Si ya se cuentan con resultados de la fenomenología de la información en las organizaciones manufactureras de dos enfoques, la disciplina administrativa y la ciencia de la información, ¿es posible complementar esos estudios?

- ¿Serán los mismos resultados en cuanto al uso de la información por parte de los directivos de las organizaciones?

- Ante el hecho de contar con resultados y conclusiones de dos disciplinas, es posible elaborar un modelo para los estudios de la información en las fábricas.

- Si ya se ha verificado la existencia de investigaciones en el lugar de trabajo de otro tipo de organizaciones, distintas a las manufactu- 
reras, en las cuales se identifican temas como el comportamiento informativo y su medición, la creación de comunidades de práctica, o en su defecto la administración de información por parte de trabajadores móviles, ¿es posible aplicar esas bases teóricas? ¿Qué implicaciones metodológicas serían aplicadas para estudios con esas temáticas en las fábricas?

\section{Conclusiones}

De los escasos estudios encontrados en un periodo de cincuenta años en las fuentes de información investigadas, se infiere que hay muchas posibilidades de continuar con el estudio de los flujos de información en las fábricas. Los estudios de los flujos de información en las organizaciones manufactureras no son exclusivos en los países desarrollados; hay un interés por estos en los países emergentes.

Las aportaciones de la disciplina administrativa para el conocimiento de los flujos de información en la estructura de las organizaciones manufactureras son, principalmente, las siguientes: a) la cobertura de la información se extiende en todas sus áreas: la gerencia, administración, producción y ventas; b) el uso de la información se concentra principalmente en la gerencia y la administración; c) la información puede generar innovación en las organizaciones; d) los gerentes y administradores compilan información respecto a sus clientes, proveedores y competencia; e) los administradores de las organizaciones manufactureras crean y se apoyan en herramientas informativas para la toma de decisiones; y f) las personas se convierten en fuente en parte integral de los flujos de información.

Aunque hay una alta concentración de estudios de la información en el área administrativa de las organizaciones manufactureras, se abre un compás de oportunidades para conocer lo que sucede en los otros espacios de este tipo de organizaciones.

\section{Notas}

(1) Se encontró otra investigación de Kock \& McQueen (1998), quienes describen cómo el conocimiento y la información son comunicados en diferentes tipos de procesos en organizaciones diversas. Sin embargo, no se incluye en la presentación en resultados, porque se ha incluido a sujetos de investigación no homogéneos, es decir, se analizan tanto organizaciones manufactureras de autopartes, como universidades e instituciones gubernamentales.

(2) Hay otra investigación que versa sobre la manera en que se comparte la información y la respuesta de las redes de proveedores para mejorar la capacidad en el tiempo de venta de productos. Sin embargo, no se incluyó en este análisis porque no menciona el país de los sujetos seleccionados ni el tamaño de las organizaciones manufactureras. El estudio tiene como sujetos de estudio a 294 responsables de la industria en el área de proveedores (Thatte, Muhammed \& Agrawa, 2008).

(3) Desde el enfoque de la ciencia de la información, el hecho de investigar distintos tipos de organizaciones es analizado en Martínez Musiño (2011a, 2011b).

(4) Se ha incluido en esta investigación el artículo de Dimitriadis \& Koh (2005) porque, aunque no indica que los sujetos de estudio sean específicamente organizaciones manufactureras, los investigadores señalan, en la parte metodológica, que en esta región se concentra el 85 por ciento de empleos para la industria. Por otra parte, la revista en la que se publica este artículo se especializa en producción, planeación y control.

\section{Referencias}

Albaum, G. (1964). Horizontal information flow: an exploratory study. // Academy of Management Journal. 7:1 (1964) 21-33.

Albaum, G. (1967). Information flow and decentralized decision making in marketing. // California Management Review. 9:4 (1967) 59-70.

Almeida Moraes, Giseli Diniz de; Escrivão Filho, E. (2006). A gestão da informação diante das especificidades das pequenas empresas. // Ciência da informação. 35:3 (2006) 124-132.

Baker, N. R.; Freeland, J. R. (1972). Structuring information flow to enhance innovation. // Management Science, 19:1 (1972) 105-116.

Bartol, K. M.; Martin, D. C. (1998). Management. (3a ed.). Boston: McGraw-Hill, 1998.

Bergeron, P. (1997). A qualitative case study approach to examine information resources management. // Canadian Journal of Information and Library Science. 22:3/4 (1997) 1-19.

Chan, S. Y. (2001). The use of graphs as decision aids in relation to information overload and managerial decision quality. // Journal of Information Science. 27:6 (2001) 417-425.

Choo, C. W.; Bergeron, P.; Detlor, B.; Heaton, L. (2008). Information culture and information use: An exploratory study of three organizations. // Journal of the American Society for Information Science and Technology. 59:5 (2008) 792-804.

Daft, R. L.; Marcic, D. (2006). Introducción a la administración (4a ed.). Australia: Thomson, 2006.

De Saulles, M. (2007). Information literacy amongst UK SMEs: An information policy gap. // Aslib Proceedings: New Information Perspectives. 59:1 (2007) 68-79.

Dimitriadis, N. I.; Koh, S. C. L. (2005). Information flow and supply chain management in local production networks: the role of people and information systems. // Production Planning \& Control. 16:6 (2005) 545-554.

Gabarro, J. J.; Kotter, J. P. (1992). Managing your boss. // Gabarro, J. J. (Ed.), Managing people and organizations. Boston, Mass.: Harvard Business School Publications. 1992. 227-238

Goldman, A. S. (1959). Information flow and worker productivity. // Management Science. 5:3 (1959) 270-278.

Higgins, J. M. (1991). The management challenge: an introduction to management. New York; Toronto: MacmiIlan/Collier Macmillan Canada, 1991.

Kinicki, A.; Williams, B. K. (2006). Management: a practical introduction. (2a ed.). Boston: McGraw-Hill, 2006. 
Jones, G. R.; George, J. M. (2008). Contemporary management. (5a ed.). Boston: McGraw-Hill//rwin, 2008.

Jones, G. R.; George, J. M.; Hill, C. W. L. (2000). Contemporary management. (2a ed.). Boston: McGraw-Hill/lrwin, 2000.

Kock, N.; McQueen, R. (1998). Knowledge and information communication in organizations: an analysis of core, support and improvement processes. // Knowledge and Process Management. 5:1 (1998) 29-40.

Koontz, H.; O'Donell, C. (1970). Curso de administración moderna: un análisis de las funciones de la administración. $3^{\mathrm{a}}$ ed. México: McGraw-Hill, 1970.

Kotter, J. P. (1984). ¿Qué hacen realmente los gerentes generales eficientes? // Biblioteca Harvard de Administración de Empresas. 375 (1984) 1-18.

Kristiansen, S.; Kimeme, A.; Wahids, F. (2005). Information flow and adaptation in Tanzania cottage industries. // Entrepreneurship and Regional Development. 17:5 (2005) 355-388.

Kudyba, S. (2006). Enhancing organizational information flow and knowledge creation in re-engineering supply chain systems: an analysis of the U.S. automotive parts and supplies model. // International Journal of Information Management. 10:2 (2006) 163-173.

Martínez Musiño, C. (2011a). Las investigaciones de la fenomenología de la información en las fábricas: análisis de su metodología. // Crítica Bibliotecológica. 4:2 (2011) 33-44. http://eprints.rclis.org/bitstream/10760/16986/1/cb vol.4.no.2.martinez.pdf

Martínez Musiño, C. (2011b). Tipificación y uso de los recursos de información en las organizaciones manufactureras. // Scire: Representación y organización del conocimiento. 17:1 (2011) 93-100. http://ibersid.eu/ojs/index. $\mathrm{php} / \mathrm{scire} /$ article/download/3914/3634

Martínez Musiño, C. (2012a). La información y sus revoluciones: liderazgo de Peter F. Drucker. // XLII Jornadas Mexicanas de Biblioteconomia: memorias, 4,5 y 6 de mayo de 2011. San Luis Potosí, S.L.P.: Asociación Mexicana de Bibliotecarios, 2012. 475-485.

Martínez Musiño, C. (2012b). Los flujos de información en las organizaciones: análisis de los enfoques de la admi- nistración y la ciencia de la información. México: C. Martínez Musiño, 2012. 156 p. Tesis (Doctorado):Facultad de Filosofía y Letras, Instituto de Investigaciones Bibliotecológicas y de la Información, UNAM.

Martínez Musiño, C. (2013). La información en la disciplina administrativa. // E-Ciencias de la información. 3:2 (2013) 1-15. http://revistaebci.ucr.ac.cr/volumenes/3/32/3-2-3/3-2-3.pdf

Mbambo, B.; Cronjé, J. C. (2002). The internet as an information conduit in developing countries: An investigation of World Wide Web usability among small and medium textile enterprises in Botswana. // ASLIB Proceedings: New Information. 54:4 (2002) 251-259.

Mintzberg, H. (1992). The manager's job: folklore and fact. // Gabarro, J. J. (Ed.), Managing people and organizations. Boston, Mass.: Harvard Business School Publications.Management, 1992. 13-32

Moving and using information in your organization (2006). The Manager's Electronic Resource Center. [USA]: Management Sciences for Health, 2006. http://erc.msh.org/ mainpage $. \mathrm{cfm} ? \mathrm{file}=2.2 .60 . \mathrm{htm} \&$ module $=$ info\&language $=$ English.

Peterson, E. R. (2004). Seven revolutions: global strategic trends out to the year 2025. // Multinational Business Review. 12:2 (2004) 111-119.

Robbins, S. P. (2000). Managing today! (2a ed.). Upper Saddle River, N.J.: Prentice Hall, 2000.

Thatte, A. A.; Muhammed, S.; Agrawal, V. (2008). Effect of information sharing and supplier network responsiveness on time-to-make capability of a firm. // Review of Business Research. 8:2 (2008) 118-131.

Verville, J.; Halingten, A. (2003). Information searches: A two-dimensional approach for ERP acquisition decision. // Journal of Information Science. 29:3 (2003) 203-209.

Enviado: 2012-08-25. Segunda versión: 2013-07-16. Aceptado: 2013-08-25. 